BULL. AUSTRAL. MATH. SOC.

VOL. $22(1980), 339-364$.

\title{
CONJUGACY CLASSES IN PROJECTIVE AND SPECIAL LINEAR GROUPS
}

\author{
G.E, WALL
}

The conjugacy classes in the finite-dimensional projective full linear, special linear and projective special linear groups over an arbitrary commutative field are determined. The results over a finite field are applied to certain enumerative problems.

\section{Introduction}

One of the first things to establish about a given group is the distribution of its elements into conjugacy classes. In the case of the full linear group $G L_{n}(F)$, where $F$ is a (commutative) field, this information is supplied by the classical theory of the similarity of matrices. The object of the present paper is to develop the corresponding theory for the groups $P G L_{n}(F), S L_{n}(F)$ and $P S L_{n}(F)$. The methods are direct and elementary, keeping within the usual framework of simiiarity theory. Special attention is paid to the case of a finite coefficient field, where the results take a particularly simple and transparent form. The fact that the special and projective indices (defined in (3.17) and (3.18)) enter the relevant formulae in a symmetrical way is the source of the dualities observed by Lehrer ([4], Theorem B) and Macdonald ([5], Remark after (4.6)).

Macdonald ([5]) also develops the conjugacy theory over finite fields, although by somewhat different methods (for example, greater emphasis is placed on a certain partition of $n$ called the type). Reading his paper

Received 29 April 1980. 
stimulated me to work out several further results, which appear here as $(4.17)-(4.21)$.

\section{General principles}

Let $F$ be a (commutative) field. Denote by $F^{*}$ the multiplicative group of its non-zero elements. We consider the full linear group $G L_{n}(F)$. The homomorphism

$$
\operatorname{det}: G L_{n}(F) \rightarrow F^{*}
$$

maps $G L_{n}(F)$ onto $F^{*}$ and has kernel $S L_{n}(F)$, so that $G L / S L \cong F^{*}$.

Hence, if $G$ is a subgroup of $G L$,

$$
G / S G \cong \operatorname{det} G
$$

where

$$
S G=G \cap S L
$$

The non-zero scalar matrices $\lambda I$ form a central subgroup $Z=Z(n, F)$ of $G L$ isomorphic to $F^{*}$. The canonical homomorphism

$$
P: G L \rightarrow P G L=G L / Z
$$

carries each subgroup $G$ of $G L$ onto its projective counterpart $P G=G Z / 2$.

The subgroup $G$ acts by conjugation on $G L$. The G-class of a nonsingular matrix $A$ is defined as its orbit under this action, namely,

$$
\text { (2.I) } \quad(A)_{G}=\left\{T A T^{-1}: T \in G\right\} \text {. }
$$

The G-classes of elements of $G$ are just the conjugacy classes of $G$.

Similarly, $P G$ acts by conjugation on $P G L$. The $P G$-class of a nonsingular matrix $A$ is defined to be

$$
\text { (2.2) } \quad(A)_{P G}=\left\{\lambda\left(T_{A T}{ }^{-1}\right): \lambda \in F^{*}, T \in G\right\} \text {. }
$$

In other words,

$$
(A)_{P G}=P^{-1} \quad \text { (orbit of } P A \text { under } P G \text { ). }
$$

so that there is a canonical one-one correspondence between $P G$-classes and 
orbits under the action of $P G$ on $P G L$.

Let us now compare ${ }^{(A)_{G}},(A)_{S G},(A)_{P G}$ and $(A)_{P S G} \cdot$ For this purpose we introduce the following groups:

$$
\begin{aligned}
& C=C_{G}(A)=\left\{T \in G: T A T^{-1}=A\right\}, \\
& \Gamma=\Gamma_{G}(A)=\left\{T \in G: T A T^{-1}=\text { scalar multiple of } A\right\}, \\
& L=L_{G}(A)=\left\{\lambda \in F^{*}:(\lambda A)_{G}=(A)_{G}\right\}, \\
& \Lambda=\Lambda_{G}(A)=L_{S G}(A) .
\end{aligned}
$$

We shall apply again and again the simple principle that the elements in the orbit of a given point correspond one-one to the left cosets of the stabilizer of that point.

First, $G$ acts by conjugation on the set of all $S G$-classes and $(A)_{G}$ is the union of the $S G$-classes in the orbit of $(A)_{S G}$. The stabilizer of $(A)_{S G}$ is clearly $(S G) C$. In view of the isomorphism $G /(S G) C \cong \operatorname{det} G / \operatorname{det} C$, we have:

(2.7) the SG-classes into which $(A)_{G}$ splits correspond one-one to the elements of $\operatorname{det} G / \operatorname{det} C$.

A similar argument gives:

(2.8) the PSG-classes into which $(A)_{P G}$ splits correspond one-one to the elements of $\operatorname{det} G / \operatorname{det} \Gamma$.

Next, $F^{*}$ acts on the set of all $G$-classes by the rule

$$
\lambda \circ(A)_{G}=(\lambda A)_{G} \text {, }
$$

and $(A)_{P G}$ is the union of the $G$-classes in the orbit of $(A)_{G}$. Since the stabilizer of $(A)_{G}$ is $L$, we have:

(2.10) the G-classes into which (A) ${ }_{P G}$ splits correspond one-one to the elements of $F^{*} / L$.

Replacing $G$ by $S G$ in (2.10), we get: 
(2.II) the SG-classes into which (A) ${ }_{P S G}$ splits correspond one-one to the elements of $F^{*} / \Lambda$.

Some simple properties of the groups $C, \Gamma, L$ and $\Lambda$ corresponding to a given non-singular matrix $A$ may be noted. If $T \in \Gamma$, there exists $\lambda \in F^{*}$ such that

$$
T A T^{-1}=\lambda A
$$

Taking determinants, we deduce that

(2.13) every element of $L$ is an nth root of unity; thus $L$ is a finite cyclic group of order dividing $n$.

Again, the mapping

$$
\text { mult }: \Gamma \rightarrow F^{*}
$$

which assigns to each $T \in \Gamma$ the multiplier $\lambda$ in (2.12) is a homomorphism with image $L$ and kernel $C$, so that

$$
\Gamma / C \cong L
$$

This implies that $\Gamma /(S \Gamma) C \cong L / \Lambda$. Since also $\Gamma /(S \Gamma) C \cong \operatorname{det} \Gamma / \operatorname{det} C$, we have $\operatorname{det} \Gamma / \operatorname{det} C \cong L / \Lambda$.

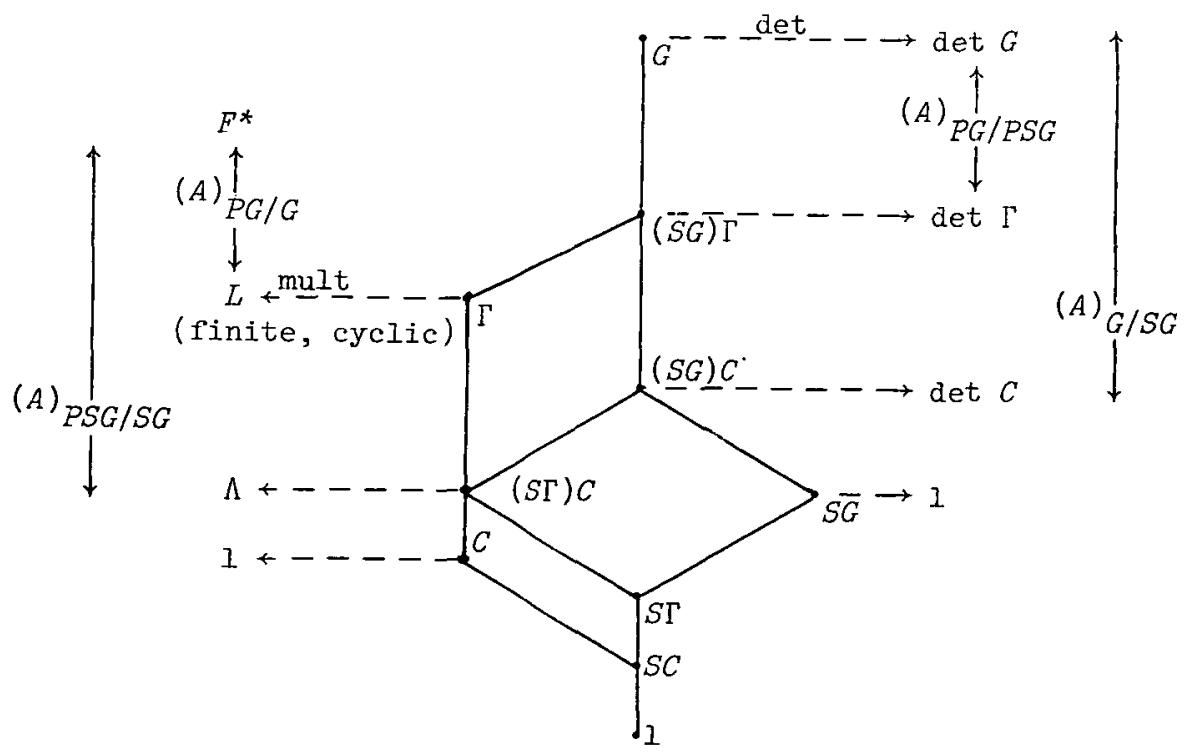

Figure 1 
Figure 1 is a lattice diagram in which meets and joins are indicated. The broken arrows to left and right show the effect of the homomorphisms mult and det. The label $(A)_{P G / G}$ (for example) indicates that the splitting of $(A)_{P G}$ into $G$-classes is governed by the quotient group $F^{*} / L$ in accordance with (2.10).

\section{The full linear group}

We now specialize to the case $G=G L_{n}(F)$. We shall determine explicitly the groups $L=L_{G L}(A), \Lambda=\Lambda_{G L}(A), \operatorname{det} C=\operatorname{det} C_{G L}(A)$ and $\operatorname{det} \Gamma=\operatorname{det} \Gamma_{G L}(A)$, where $A \in G L_{n}(F)$. The term GL-class will mean a $G L_{n}(F)$-class for some (usually unspecified) $n$. In other words, a $G L-c l a s s$ is just a similarity class of non-singular matrices over $F$. The $G L-c l a s s$ of $A$ is denoted by $(A)_{G L}$. We define $S L-, P G L-$ and $P S L$-classes in the obvious way and use the corresponding notation $(A)_{S L}$, (A) $P G L,(A)_{P S L}$

The similarity class of an $n \times n$ matrix $A$ over $F$ is determined by the elementary divisors of $A$. However, while this specification is adequate for some of our purposes, the following variant is more convenient for others. Each elementary divisor of $A$ is a power of a monic irreducible polynomial over $F$. For a given positive integer $r$, let those elementary divisors of $A$ which are $r$ th powers of monic irreducible polynomials be

$$
\pi_{1}(x)^{r}, \pi_{2}(x)^{r}, \cdots,
$$

each elementary divisor being written down with correct multiplicity. Write

$$
f_{r}(x)=\pi_{1}(x) \pi_{2}(x) \ldots
$$

Then the elementary divisors of $A$, and hence also its similarity class, are uniquely determined by the sequence

$$
\sigma(A)=\left(f_{1}(x), f_{2}(x), \ldots\right) .
$$

Macdonald [5] uses essentially the same specification of the similarity 
classes.

Note that

$$
\begin{aligned}
n(A) & =\operatorname{deg} f_{1}+2 \operatorname{deg} f_{2}+\ldots, \\
\operatorname{det} A & =\delta\left(f_{1}\right) \delta\left(f_{2}\right)^{2} \ldots,
\end{aligned}
$$

where $n(A)=n$ and $\delta(f)$ denotes the product of the roots of $f$. In particular, $A$ is non-singular if, and only if, no $f_{p}(x)$ is divisible by $x$.

From now on, we assume that $A$ is non-singular. The groups $\operatorname{det} C, L$ and det $\Gamma$ are determined in succession in Theorems 1,2 and 3 . Since $L$ is a finite cyclic group, its subgroup $\Lambda$ is then determined by the general isomorphism (2.14). Finally, in Theorem 4, these results are further specialized to the case where $F$ is a finite field.

The following result will be required in the proof of Theorem 1 .

LEMMA. Let $X \in G L_{n}(R)$, where $R$ is a commutative local ring with nilpotent maximal ideal. Then there exist products of $n \times n$ unipotent matrices $P, Q$ over $R$ such that $P X Q$ is diagonal.

Proof. Let $J$ be the maximal ideal, and $\bar{R}=R / J$ the residue class field, of $R$. Let $M_{n}(R) \rightarrow M_{n}(\bar{R}), Y \mapsto \bar{Y}$, denote the homomorphism induced by the canonical homomorphism $R \rightarrow \bar{R}$. An $n \times n$ matrix is called elementary if all its diagonal elements are $l$ and all except one of its off-diagonal elements are 0 . Since $\bar{X} \in G L_{n}(\bar{R})$ and $\bar{R}$ is a field, there exist products of elementary matrices $P_{1}, Q_{1}$ over $R$ such that $\bar{P}_{1} \bar{X} \bar{Q}_{1}=\bar{D}$, where $D$ is diagonal. Thus, $P_{1} X Q_{1}=D+Y$, where $Y \in M_{n}(J)$. Since $\bar{D}$ is non-singular, every diagonal element of $D$ lies in $R-J$ and so is a unit; hence $D \in G L_{n}(R)$ and $P_{1} X Q_{1}=D(I+Z)$, where $Z=D^{-1} Y$. But $Z \in M_{n}(J)$ and $J$ is nilpotent, so that $Z$ is nilpotent and thus $I+Z$ unipotent. Therefore $P X Q=D$, where $P=P_{1}$ and $Q=Q_{1}(I+Z)^{-1}$ are both products of unipotent matrices. This completes the proof. 
Notation. (a) If $H$ is a subgroup of $F^{*}$ and $d$ a positive integer, then

$$
\begin{aligned}
& H^{d}=\left\{h^{d}: h \in H\right\}, \\
& H_{d}=\left\{h: h \in H, h^{d}=1\right\} .
\end{aligned}
$$

(b) If $\pi$ is an irreducible polynomial in $F[x]$ and $K$ the field obtained by adjoining a root of $\pi$ to $F$, then $\Delta(\pi)$ denotes the image of $K^{*}$ under the norm homomorphism $N_{K / F}: K^{*} \rightarrow F^{*}$.

THEOREM 1. Let $A \in G L_{n}(F)$, where $F$ is a fiezd. Then

$$
\operatorname{det} C_{G L}(A)=\prod \Delta(\pi)^{r} \text {, }
$$

where the product is taken over the elementary divisors $\pi^{r}$ of $A$.

The proof will be carried out in terms of linear transformations rather than matrices. Let $T$ be a non-singular linear transformation on a finite-dimensional vector space $V$ over $F$. We turn $V$ into an $E[x]-$ module in the usual way by defining $f(x) v=f(T) v$. Let $E$ denote the ring of module endomorphisms of $V$ and $E^{*}$ the group of units of $E$. Then the assertion of the theorem is that

$$
\operatorname{det} E^{*}=\prod \Delta(\pi)^{r}
$$

where the product is taken over the elementary divisors $\pi^{r}$ of $T$.

We begin with the simplest case of ail, where $T$ has a single irreducible elementary divisor $\pi$ with multiplicity 1 . Let $K$ be the field obtained by adjoining a root $\alpha$ of $\pi$ to $F$. Then $K$ is a finite-dimensional vector space over $F$ and for each $\beta \in K$ the mapping $\hat{\beta}: K \rightarrow K, u \mapsto B u$, is $F$-linear. We may take $V=K$ and $T=\hat{\alpha}$, and it is easy to see that $E$ consists of the $\hat{\beta}$. The well-known formula $\operatorname{det} \hat{\beta}=N_{K / F}(\beta)$ now gives $\operatorname{det} E^{*}=\Delta(\pi)$, as required.

We now proceed to the next simplest case, where $T$ has a single elementary divisor $\pi^{r}$ with multiplicity 1 . Since $V$ is a cyclic module, the elements of $E$ are the polynomials $f(T)$. Let $\bar{T}$ be the linear transformation induced by $T$ on the quotient module $\bar{V}=V / \pi(x) V$ 
and let $\bar{E}$ be the ring of module endomorphisms of $\bar{V}$. Then $\bar{T}$ has the single elementary divisor $\pi$ with multiplicity $1, \bar{E}$ consists of the polynomials $f(\bar{T})$ and, by what we have already proved, $\operatorname{det} \bar{E}^{*}=\Delta(\pi)$.

We observe now that

$$
V \supset \pi(x) V \supset \ldots \supset \pi(x)^{r} V=\{0\}
$$

is a composition series for the module $V$ in which all quotient modules

$$
\pi(x)^{i-1} V / \pi(x)^{i} V \quad(i=1, \ldots, r)
$$

are isomorphic. (Indeed, multiplication by $\pi(x)^{i-1}$ gives an isomorphism of $V / \pi(x) V$ onto $\pi(x)^{i-1} V / \pi(x)^{i} V$.) It follows that

$$
\operatorname{det} f(T)=(\operatorname{det} f(\bar{T}))^{r} \text {, }
$$

whence

$$
\operatorname{det} E^{*}=\left(\operatorname{det} \bar{E}^{*}\right)^{r}=\Delta(\pi)^{r} \text {, }
$$

as required.

We turn now to the general case. Write $M\left(\pi^{r}\right)$ for the indecomposable $F[x]$-module $F[x] / \pi(x)^{r} F[x]$. Let the elementary divisors of $T$ be $\pi_{I}^{r_{1}}, \ldots, \pi_{k}^{r_{k}}$ with respective multiplicities $m_{1}, \ldots, m_{k}$. Then we may assume that

$$
V=V_{1} \oplus \ldots \oplus V_{m}\left(m=\sum m_{i}\right)
$$

where

$$
\begin{array}{r}
V_{1}=\ldots=V_{m_{1}}=M\left(\pi_{1}^{r_{1}}\right), \\
V_{m_{1}+1}=\ldots=V_{m_{1}+m_{2}}=M\left(\pi^{{ }^{2}}\right),
\end{array}
$$

and so on. In view of what we have proved already, (3.7) can be rewritten as

$$
\operatorname{det} E^{*}=\prod_{i=1}^{m} \operatorname{det} E_{i}^{*} \text {, }
$$


where $E_{i}$ is the ring of module endomorphisms of $V_{i}$.

In the present paragraph we take advantage of the direct decomposition (3.8) to identify $E$ with the ring of all $m \times m$ matrices $S=\left(s_{i j}\right)$, where $s_{i j} \in \operatorname{Hom}_{F[x]}\left(V_{i}, V_{j}\right)$ for all $i, j$. Such a matrix $S$ can be written as a $k \times k$ block matrix $\left(s_{\lambda \mu}\right)$, where $S_{\lambda \mu}$ is an $m_{\lambda} \times m_{\mu}$ matrix for all $\lambda, \mu$. Notice that

$$
S_{\lambda \lambda} \in M_{m_{\lambda}}\left(R\left({ }^{r}{ }_{\lambda} \lambda\right)\right),
$$

where

$$
R\left({ }^{r}{ }_{\lambda} \lambda\right)=\text { End } M\left({ }^{r} \lambda \lambda\right)
$$

is isomorphic to the (local) ring $F[\dot{x}] / \pi_{\lambda}(x)^{r} \lambda_{F[x]}$. We introduce the block diagonal matrix

$$
S^{\prime}=\operatorname{diag}\left(S_{11}, \ldots, S_{k k}\right)
$$

Then (see Jacobson [2], Chapter 4, Theorem 8)

$$
S^{\prime} \equiv S(\bmod \operatorname{rad} E) \text {. }
$$

Suppose now that $S$ is invertible. Then $S^{\prime}=S(I+N)$, where $N \in \operatorname{rad} E$ and so $I+N$ is unipotent. In particular, $S^{\prime}$ is invertible and so each of its diagonal blocks $S_{\lambda \lambda}$ is invertible. Applying the lemma to each of these diagonal blocks, we deduce that there exist products of (block diagonal) unipotent matrices $P_{1}, Q_{1}$ such that $P_{1} S^{\prime} Q_{1}=D$, where $D$ is diagonal. Then

$$
P S Q=D,
$$

where $P=P_{1}$ and $Q=(I+N) Q_{1}$ are also products of unipotent matrices.

Let us now regard the elements of $E$ once more as linear transformations on $V$. Then (3.10) implies that every element of $E^{*}$ has the same determinant as some element of $E^{*}$ which maps every $V_{i}$ onto itself. Since the determinants of the latter elements of $E^{*}$ obviously form the 
group $T$ det $E_{i}^{*}$, our result (3.9) follows. This completes the proof of the theorem.

Consider the set $M$ of all monic polynomials $f(x) \in F[x]$ which are not divisible by $x$. Then $F^{*}$ acts on $M$ by the rule

$$
(\lambda \circ f)(x)=\lambda^{m} f\left(\lambda^{-1} x\right) \quad(m=\operatorname{deg} f) .
$$

It is easily verified that if $f, g, \ldots$ are the elementary divisors of $A$ then $\lambda \circ f, \lambda \circ g, \ldots$ are those of $\lambda A$. Hence, in the notation (3.1),

$$
\sigma(\lambda A)=\left(\lambda \circ f_{1}, \lambda \circ f_{2}, \ldots\right)
$$

THEOREM 2. Let $A \in G L_{n}(F)$, where $F$ is a field, and let (3.1) be the corresponding sequence of polynomials. Then

$$
L_{G L}(A)=\left(F^{*}\right)_{\delta},
$$

where $\delta$ is the greatest positive integer such that $f_{r}(x) \in F\left[x^{\delta}\right]$ for alz $r$.

Proof. By (2.13), $L$ is finite. Let $\varepsilon$ be a primitive $d$ th root of unity in $F$. By (3.12), $\varepsilon \in L$ if, and only if,

(a) $\varepsilon \circ f_{r}=f_{r}$ for all $r$.

We prove the theorem by showing that (a) is equivalent to

(b) $f_{r}(x) \in F\left[x^{d}\right]$ for all $r$.

Now, $f_{r^{\prime}}(x)$ has the form $x^{m}+a_{1} x^{m-1}+\ldots+a_{m}$, where $a_{m} \neq 0$.

The equation $\varepsilon \circ f_{p}=f_{r}$ means that $a_{t}=\varepsilon^{t} a_{t}$ for all $t$ and thus that $a_{t}=0$ except when $d \mid t$. However, since $a_{m} \neq 0$, this is equivalent to $f_{r}(x) \in F\left[x^{d}\right]$. Thus, $(a)$ and $(b)$ are equivalent and the theorem is proved.

THEOREM 3. Let $A \in G L_{n}(F)$, where $F$ is a fiezd. Then $\operatorname{det} \dot{\dot{\Gamma}}_{G L}(A)$ is generated by $\operatorname{det} C_{G L}(A)$ and $(-1)^{n(n-1) / Z}$, where $z=\left|L_{G L}(A)\right|$. 
Proof. We have

$$
(-1)^{n(n-1) / 2}=\varepsilon^{\left(\begin{array}{c}
n \\
2
\end{array}\right)} \text {, }
$$

where $\varepsilon$ is a primitive 2 th root of unity in $F$. The theorem will be established by proving the existence of a matrix $T$ over $F$ such that

$$
T A T^{-1}=\varepsilon A, \quad \operatorname{det} T=\varepsilon^{\left(\begin{array}{l}
n \\
2
\end{array}\right)} .
$$

Consider an elementary divisor $h_{1}$ of $A$. Let $h_{1}, \ldots, h_{s}$ be the distinct members of its orbit under the action of $L=L_{G L}(A)$ given by (3.11). Since $\varepsilon \in L, A$ is similar to $\varepsilon A$ and so all $h_{i}$ have the same multiplicity as elementary divisors of $A$. Since the $h_{i}$ are relatively prime in pairs, the direct sum of the companion matrices of the $h_{i}$ is similar to the companion matrix of their product $h=h_{1} \ldots h_{s}$.

Clearly, $h(x)$ has the form $g\left(x^{2}\right)$, where $g(x) \in F[x]$. These considerations show that we may assume that $A$ is the block diagonal matrix $\operatorname{diag}\left(A_{1}, \ldots, A_{t}\right)$, where each $A_{r}$ is the companion matrix of a polynomial $g_{r}\left(x^{2}\right)$ with $g_{r}(x) \in F[x]$.

Suppose that, for each $r$, we have found a matrix $T_{r}$ such that

$$
{ }{ }_{r}{ }_{r} T_{r}^{-1}=\varepsilon A_{r}, \quad \operatorname{det} T_{r}=\varepsilon^{\left(\begin{array}{c}
n_{r} \\
2
\end{array}\right)},
$$

where $A_{p} \in G L_{n_{p}}(F)$. Then the block diagonal matrix $T=\operatorname{diag}\left(T_{1}, \ldots, T_{t}\right)$ satisfies $T A T^{-1}=\varepsilon A$ and

$$
\operatorname{det} T=\prod \varepsilon^{\left(\begin{array}{c}
n \\
r \\
2
\end{array}\right)}=\varepsilon^{\left(\begin{array}{c}
n \\
2
\end{array}\right)},
$$

since $n=\sum n_{r}$ and each $n_{r}$ is divisible by $l$. Thus, it is sufficient to establish the existence of a matrix $T$ satisfying (3.14) when $A$ itself is the companion matrix of a polynomial $f(x)=g\left(x^{2}\right)$, where 
$g(x) \in F[x]$

Let $K$ be the commutative ring obtained by adjoining a root $\alpha$ of $f(x)$ to $F$. Then $K$ is an n-dimensional vector space over $F$ with basis $1, \alpha, \ldots, \alpha^{n-I}$. The mapping $\hat{\alpha}: K \rightarrow K, u \mapsto \alpha u$, is an $F$-linear transformation and its matrix with respect to the above basis is $A$. It is therefore sufficient to prove that $\hat{\alpha}$ is similar to $\varepsilon \hat{\alpha}$ by a linear transformation $\tau$ of determinant $\varepsilon^{\left(\begin{array}{c}n \\ 2\end{array}\right)}$. Now, since $f(x) \in F\left[x^{2}\right]$, there is an automorphism of $K$ which carries $\alpha$ to $\varepsilon \alpha$ and fixes the elements of $F$. We take $\tau$ to be this automorphism. Since $\tau\left(\alpha^{i}\right)=\varepsilon^{i} \alpha^{i}$, the determinant of $\tau$ is

$$
\varepsilon^{1+2+\ldots+(n-1)}=\varepsilon^{\left(\begin{array}{l}
n \\
2
\end{array}\right)},
$$

as required. This completes the proof.

COROLLARY. The quotient group $\operatorname{det} \Gamma_{G L}(A) / \operatorname{det} C_{G L}(A)$ has order 1 or 2 . It has order 2 if, and only if, $-1 \notin \operatorname{det} C_{G L}(A)$ and $n / 2$ is odd.

Proof. Theorem 3 shows at once that if $-1 \in \operatorname{det} C$ then $\operatorname{det} C=\operatorname{det} \Gamma$. Suppose therefore that $-1 \neq \operatorname{det} C$. By (2.13), $n / 2$ is an integer. If $n / 2$ is even, then $(-1)^{n(n-1) / 2}=1$ and so, by Theorem 3 , $\operatorname{det} C=\operatorname{det} \Gamma$. Suppose therefore that $n / 2$ is odd. Then $(-1)^{n(n-1) / 2}=(-1)^{n-1}$. Since $-I \in C$ but $-1 \notin \operatorname{det} C$, it follows that $n$ is even and thus that $(-1)^{n-1}=-1$. Since $-1 \notin \operatorname{det} C$, Theorem 3 now shows that $\operatorname{det} C$ has index 2 in $\operatorname{det} \Gamma$. This completes the proof.

We have just shown that the quotient group $\operatorname{det} \Gamma / \operatorname{det} C$ has order 1 or 2 . The following example shows that both values are possible. Recall that $\operatorname{det} \Gamma /$ det $C \cong L / \Lambda$.

EXAMPLE. Take

$$
A=\left(\begin{array}{cc}
0 & 1 \\
-1 & 0
\end{array}\right) \in S L_{2}(F) \text {. }
$$

It is easy to see that $L=\{1,-1\}$. Now, the general solution of 
$T A=-A T$ is

$$
T=\left(\begin{array}{cc}
a & b \\
b & -a
\end{array}\right),
$$

where clearly $\operatorname{det} T=-\left(a^{2}+b^{2}\right)$. If $F=\mathbb{R}$, $\operatorname{det} T=1$ has no solution $a, b$ in $F$ and thus $\Lambda=\{1\}$. On the other hand, if $F=\mathbb{C}$, $\operatorname{det} T=1$ has a solution in $F$ and so $\Lambda=L$.

To conclude this section, we consider the case of a finite coefficient field.

THEOREM 4. Let $A \in G L_{n}\left(1 F_{q}\right)$ and let (3.1) be the corresponding sequence of polynomials. Then

$$
\begin{aligned}
\operatorname{det} C_{G L}(A) & =\operatorname{det} \Gamma_{G L}(A)=\left(\mathbb{F}_{q}^{*}\right)^{d(A),}, \\
L_{G L}(A) & =\Lambda_{G L}(A)=\left(\left[\mathbb{F}_{q}^{*}\right)_{\delta(A)},\right.
\end{aligned}
$$

where

(3.17) $d(A)$ is the greatest divisor $d$ of $q-1$ such that $f_{r}(x)=1$ whenever $r$ is not a multiple of $d$, and

(3.18) $\delta(A)$ is the greatest divisor $\delta$ of $q-1$ such that $f_{r}(x) \in F\left[x^{\delta}\right]$ for alz $r$.

Proof. In view of (2.14), it is sufficient to prove that
(a) $\operatorname{det} C=\left(E^{*}\right)^{d(A)}$,
(b) $L=\left(F^{*}\right) \delta(A)$,
(c) $\operatorname{det} C=\operatorname{det} \Gamma$,

where $F=\mathbb{F}_{q}$.

Proof of (a). Let the elementary divisors of $A$ be $\pi_{1}^{r_{1}}, \pi_{2}^{r_{2}}, \ldots$, where $\pi_{+} \pi_{2}, \ldots$ are irreducible. Then

$$
d(A)=(q-1, d),
$$


where $d$ is the greatest common divisor of $r_{1}, r_{2}, \ldots$. Moreover, since $F$ is finite, $\Delta\left(\pi_{i}\right)=F^{*}$ for all $i$. Therefore, by Theorem 1 ,

$$
\operatorname{det} C=\prod_{i}\left(F^{*}\right)^{r^{r}}=\left(F^{*}\right)^{d}=\left(E^{*}\right)^{d(A)} \text {. }
$$

Proof of (b). We have

$$
\delta(A)=(q-1, \delta),
$$

where $\delta$ is the greatest positive integer such that $f_{p}(x) \in E\left[x^{\delta}\right]$ for all $r$. By Theorem 2,

$$
L=\left(F^{*}\right)_{\delta}=\left(F^{*}\right)_{\delta(A)}
$$

Proof of (c). By the Corollary to Theorem 3, it is sufficient to prove that if $-1 \notin \operatorname{det} C$ then $n / \delta(A)$ is even. Now, if $-1 \notin \operatorname{det} C$, then $d(A)$ is even by (a). Thus, it will be sufficient to prove that

$$
d(A) \delta(A) \mid n \text {. }
$$

In the formula (3.2) for $n=n(A), \delta(A) \mid \operatorname{deg} f_{r}$ for all $r$, and $\operatorname{deg} f_{p}=0$ unless $r \mid d(A)$. Thus, all terms $r$ deg $f_{r}$ are divisible by $d(A) \delta(A)$ and (3.19) follows. This proves the theorem.

COROLLARY. If $A \in G L\left(n, \mathbb{F}_{q}\right)$, then

$(3.20)$

$$
\left\{\begin{array}{l}
{ }_{G L}^{(A)} \text { splits into } d(A) \text { SL-classes, } \\
(A)_{P G} \text { splits into } d(A) \text { PSL-classes, } \\
(A)_{P G L} \text { splits into }(q-1) / \delta(A) \text { GL-classes, } \\
(A)_{P S L} \text { splits into }(q-1) / \delta(A) \text { SL-classes. }
\end{array}\right.
$$

Proof. This follows at once from the theorem and (2.7), (2.8), $(2.10),(2.11)$.

\section{Enumeration, duality}

In this section, $F$ will be the finite field $\mathrm{IF}_{q}$. Let $A \in G L_{n}=G L_{n}(F)$. With (3.20) in mind, we call $d(A)$ the special index, 
or s-index, of $A$ and $\delta(A)$ the projective index, or $p$-index, of $A$. By (3.17)-(3.19), both are divisors of $q-1$ and their product is a divisor of $n$. Since all matrices in the one PGL-class have the same $s-$ and $p$-indices, we may speak of the $s-$ and $p$-indices of a PGL-class or of any subset such as a GL class.

We begin with a detailed discussion of the results to be proved. Write

$$
C(t)=\prod_{r=1}^{\infty}\left(\left(1-t^{r}\right) /\left(1-q t^{r}\right)\right) .
$$

Feit and Fein [1] showed that

$$
c(t)=1+\sum_{n=1}^{\infty} c_{n} t^{n},
$$

where $c_{n}$ is the number of conjugacy classes in $G L_{n}$. Our first result is that, if $d$ and $\delta$ are divisors of $q-1$,

$$
1+\sum_{n=1}^{\infty} r_{n}(d, \delta) t^{n}=c\left(t^{d \delta}\right),
$$

where $\gamma_{n}(d, \delta)$ is the number of conjugacy classes in $G L_{n}$ with s-index divisible by $d$ and $p$-index divisible by $\delta$. In other words, if $d$ and $\delta$ are divisors of $q-1$,

$$
\gamma_{n}(d, \delta)= \begin{cases}c_{n / d \delta} & \text { if } d \delta \mid n, \\ 0 & \text { otherwise. }\end{cases}
$$

This result enables us to determine the number, $c_{n}(d, \delta)$, of conjugacy classes in $G L_{n}$ with $s$-index $d$ and $p$-index $\delta$. Assuming still that $d$ and $\delta$ are divisors of $q-1$, we have

$$
\gamma_{n}(d, \delta)=\sum_{\substack{D, \Delta \\ d|D|_{q-1} \\ \\ \delta|\Delta| q-1}} c_{n}(D, \Delta),
$$

whence, by the Möbius inversion formula, 
(4.6)

$$
1+\sum_{n=1}^{\infty} c_{n}(d, \delta) t^{n}=\sum_{\substack{D, \Delta \\ d|D| q-1 \\ \delta|\Delta| q-1}} \mu(D / d) \mu(\Delta / \delta) C\left(t^{D \Delta}\right)
$$

More explicitly, if $d \delta$ is not a divisor of $n$, then

$$
c_{n}(d, \delta)=0 \text {, }
$$

and if $d \delta$ is a divisor of $n$,

$$
c_{n}(d, \delta)=\sum_{\substack{D, \Delta\\
} \mid \begin{array}{l}
D \mid(n, q-1) \\
\Delta \mid \\
(n \Delta, q-1) \\
\Delta \Delta \mid n
\end{array}} \mu(D / d) \mu(\Delta / \delta) c_{n / D \Delta} .
$$

In particular, we see that

$$
c_{n}(d, \delta)=c_{n}(\delta, d)
$$

This simple result is the source of the later results on duality.

The above formulae can be generalised. Let $D, E$ be divisors of $q-1$. We introduce the following subgroups of $G L_{n}$ :

$$
\begin{aligned}
& P^{D}=P^{D}(n)=\left\{A: \operatorname{det} A \in\left(E^{*}\right)^{D}\right\}, \\
& Z_{E}=Z_{E}(n)=\left\{\lambda I: \lambda \in\left(E^{*}\right)_{E}\right\} .
\end{aligned}
$$

If, in addition, $D E \ln (q-1)$, then $z_{E} \subseteq P^{D}$ and we may form the quotient group

$$
P_{E}^{D}=P_{E}^{D}(n)=P^{D} / Z_{E}
$$

Each subgroup $G$ of $G L_{n}$. acts on $P_{E}^{D}$ by conjugation. Slightly extending the notation of Section 2, we call the orbits G-classes. Then the duality theorem of Lehrer cited in the introduction asserts that if $D E \mid q-1$ then the numbers of $G L_{n}$-classes in $P_{E}^{D}(n)$ and $P_{D}^{E}(n)$ are the some.

In practice it is convenient to deal, not with the G-classes in $P_{E}^{D}$ themselves, but rather with their inverse images under the canonical 
homomorphism $P^{D} \rightarrow P_{E}^{D}$. These are the sets

$$
{ }_{E ; G} \quad\left(A \in P^{D}\right) \text {, }
$$

where

$$
(A)_{E ; G}=\left\{\lambda T A T^{-1}: \lambda \in\left(F^{*}\right)_{E}, T \in G\right\} \text {. }
$$

Since $(A)_{E ; G} \subseteq(A)_{P G L}$, we may speak of the $s-$ and $p$-indices of ${ }^{(A)}{ }_{E ; G}$ and hence of the corresponding G-class. Our second result is that (4.15) if $D, E, d, \delta, \Delta$ are divisors of $q-1$ such that $D E \mid n(q-1)$, then the number of $P^{\Delta}$-classes in $P_{E}^{D}(n)$ with $s$-index $d$ and p-index $\delta$ is

$$
\frac{(d, D)(\delta, E)}{D E}(d, \Delta) c_{n}(d, \delta)
$$

Some special cases are of interest. When $D=\Delta$, we get the number of conjugacy classes in $P_{E}^{D}(n)$ with s-index $d$ and $p$-index $\delta$. Specialising even further, we get the numbers of such conjugacy classes in $P G L_{n}, S L_{n}$ and $P S L_{n}$. The total numbers of conjugacy classes in the latter groups are discussed in detail by Macdonald in [5].

Again, taking $\Delta=1$ in (4.15) and using (4.9), we deduce that (4.16) if $d, \delta, D, E$ are divisors of $q-1$ such that $D E \mid n(q-1)$, then the number of $G L_{n}$-classes in $P_{E}^{D}(n)$ with s-index $d$ and $p$-index $\delta$ is equal to the number of $G L_{n}$-classes in $P_{D}^{E}(n)$ with s-index $\delta$ and $p$-index $d$.

This clearly implies Lehrer's theorem.

Another consequence of (4.15) is the following:

(4.17) Let $D, E, \Delta$ be divisors of $q-1$ and suppose that $D E \mid n(q-1)$. Then the total number of $P^{\Delta}$-classes in $P_{E}^{D}(n)$ is 


$$
(D E)^{-1} \sum_{\substack{d, \delta \\ \delta|E, d|[D, \Delta] \\ d \delta \mid n}} \phi\left(d_{1}\right) \phi_{2}\left(d_{2}\right) d_{3}^{2} \phi(\delta) c_{n / d \delta},
$$

where $d_{1}=d /(d, D, \Delta), d_{2}$ is the largest divisor of $d$ relatively prime to $d_{1}$ and $d_{1} d_{2} d_{3}=d$.

Here, $\phi(m)=\phi_{1}(m)$ and $\phi_{2}(m)$ are the Eulerian functions defined by

$$
\phi_{p}(m)=m^{r} \prod_{p}\left(1-\left(1 / p^{r}\right)\right)
$$

where summation is over the distinct prime divisors $p$ of $m$. We shall pass over the proof of (4.17) except to mention that it depends on summing $\sum_{d\rceil m} \mu(m / d)\left(d, m_{1}\right)\left(d, m_{2}\right)$ in closed form.

Taking $\Delta=1$ in (4.17), we get the following formula for the number of rg $_{n}$-classes in $P_{E}^{D}(n):$

$\pi \dot{I} \quad a s$

$$
(D E)^{-1} \sum_{\substack{d, \delta \\ d|D, \delta| E \\ d \delta \mid n}} \phi(d) \phi(\delta) c_{n / d \delta}
$$

It follows that, for given $n$ and $q$, the number of $G L_{n}$-classes in $P_{E}(n)$ depends only on $D E$ and $(D, E)$. This is a slight generalisation of Lehrér's theorem.

Similar results can be proved by similar methods for the groups of $F$-rational points of the connected algebraic groups isogenous to $S L_{n}(\bar{F})$, where $\bar{F}$ is the algebraic closure of $F$. These have the same order as $S L_{n}$ and include both $S L_{n}$ and $P G L_{n}$ as special cases. In the formulation (but not the notation) of Macdonald [5], they appear as the quotient groups

$$
Q_{e}=Q_{e}(n)=R_{e} / S_{e}(e \mid n),
$$

where $R_{e}^{(N)}, \mathcal{S}_{e}$ are the following subgroups of $G L_{n} \times F^{*}$ : 


$$
\begin{aligned}
& R_{e}=\left\{(X, \lambda): \operatorname{det} X=\lambda^{e}\right\}, \\
& S_{e}=\left\{\left(\alpha I, \alpha^{n / e}\right): \alpha \in F^{*}\right\} .
\end{aligned}
$$

The action of $G L_{n}$ on $Q_{e}$ is defined via the embedding $G L_{n} \rightarrow G L_{n} \times F^{*}$, $X \mapsto(X, 1)$. If $G$ is a subgroup of $G L_{n}$, the orbits under the action of $G$ on $Q_{e}$ are again called G-classes. The duality theorem of Macdonald referred to in the introduction asserts that if ef $=n$ then the numbers of $G L_{n}$-classes in $Q_{e}(n)$ and $Q_{f}(n)$ are the same. Indeed the explicit formula used to prove this result yields the slightly stronger result that if ef $=n$ then the numbers of $G L_{n}$-classes in $Q_{e}(n)$ and $Q_{(e, f, q-1)}(n)$ are the same.

The $p$ - and s-indices of the G-class of an element $(X, \lambda) S_{e}$ of $Q_{e}$ are defined as the $p$ - and s-indices of $X$. The following result is the analogue of (4.15).

(4.19) Let $d, \delta, \Delta$ be divisors of $q-1$ and $e, f$ positive integers such that $e f=n$. Then the number of $P^{\Delta}$-classes in $Q_{e}(n)$ with $s$-index $d$ and $p$-index $\delta$ is

$$
\frac{(d, e)(\delta, f)}{(q-1)}(d, \Delta) c_{n}(d, \delta) \text {. }
$$

Special cases are again of interest. When $\Delta=(e, p-1)$, the $P^{\Delta}$-classes in $Q_{e}(n)$ become the conjugacy classes of $Q_{e}(n)$. Again, taking $\Delta=1$ we get the following analogue of $(4.16)$.

(4.20) Let $d, \delta \mid q-1$ and ef $=n$. Then the number of $G L_{n}$-classes in $Q_{e}(n)$ with s-index $d$ and $p$-index $\delta$ is equal to the number of $G L_{n}$-classes in $Q_{f}(n)$ with $s$-index $\delta$ and $p$-index $d$.

Macdonald's duality theorem is an immediate consequence. Finally, we have the following analogue of $(4.17)$.

(4.21) Let $\Delta \mid q-1$ and $e f=n$. Then the total number of 
$P^{\Delta}$-classes in $Q_{e}(n)$ is

$$
\sum_{\substack{d, \delta\lceil q-1 \\ \delta|f, d|[e, \Delta] \\ d \delta \mid n}} \phi\left(d_{1}\right) \phi_{2}\left(d_{2}\right) d_{3}^{2} \phi(\delta) c_{n / d \delta},
$$

where $d_{1}=d /(d, e, \Delta), d_{2}$ is the zargest divisor of $d$ relatively prime to $d_{1}$ and $d_{1} d_{2} d_{3}=d$.

EXAMPLE. Suppose that $e|n| q-1$ and write $e f=n, m n=q-1$. Each of the groups $Q_{e}(n)$ and $P_{(q-1) / e^{(n)}}^{e}$ is an extension of $S L_{n} / Z_{f}$ by a cyclic group of order $f$. Ketter and Lehrer [3] carried out computer calculations to determine the numbers of $G L_{n}$-classes in these groups for certain $e, n$ and $q$. Now, (4.21) and (4.17) show that these numbers are, respectively,

$$
\begin{aligned}
& M_{e}=\sum_{d\left|e^{\prime}, \delta\right| f} \phi(d) \phi(\delta) c_{n / d \delta}^{\prime}, \\
& N_{e}=\sum_{d|e, \delta|(m, e / d) f} \phi(d) \phi(\delta) c_{n / d \delta}^{\prime},
\end{aligned}
$$

where $c_{p}^{\prime}=c_{p} /(q-1)$. Macdonald [5] proved the formula for $M_{e}$ and tabulated $c_{r}^{\prime}$ for $r \leq 12$. Splitting $d, \delta$ into their prime-powers, one see that

$$
M_{e}=M_{(e, f)}
$$

and so, in particular, $M_{e}=M_{f}$. Further

$$
N_{e} \geq M_{e}
$$

and, as Ketter and Lehrer observed,

$$
N_{e}=M_{e} \text { if }(m, e)=1 \text {. }
$$

For $n=4$, we have 


$$
\begin{aligned}
M_{1} & =N_{1}=M_{4}=q^{3}+q^{2}+2 q+3, \\
M_{2} & =M_{1}+q, \\
N_{2}-M_{2} & =0 \text { or } 2 \text { according as }(m, 2)=1 \text { or } 2, \\
N_{4}-M_{4} & =0, q+2 \text { or } q+4 \text { according as }(m, 4)=1,2 \text { or } 4 .
\end{aligned}
$$

Our results agree with those of Ketter and Lehrer except in one case: the values when $n=6, q=13$, should be

$$
\begin{aligned}
& M_{1}=M_{2}=M_{3}=M_{6}=N_{1}=N_{3}=402432, \\
& N_{2}=N_{6}=402616 .
\end{aligned}
$$

The proofs of the results for the groups $Q_{e}(n)$ will be omitted. It remains to prove the key results (4.3) and (4.15). In each case, some preparation is necessary.

Let $P$ be a set of monic polynomials over $F$ such that $1 \in P$. The generating function for $P$ is defined to be the power series

$$
g_{P}(t)=\sum_{n=0}^{\infty} g_{n}(P) t^{n},
$$

where $g_{n}(P)$ denotes the number of elements of $P$ of degree $n$. (Notice that $g_{0}(P)=1$ since $\left.1 \in P.\right)$ In the same way, if $Q$ is a set of similarity classes of square matrices over $F$, the generating function for $Q$ is the power series

$$
G_{Q}(t)=1+\sum_{n=1}^{\infty} G_{n}(Q) t^{n},
$$

where $G_{n}(Q)$ denotes the number of similarity classes of $n \times n$ matrices in $Q$. The following enumerative principle is due to Feit and Fein [1].

LEMMA. Given sets $P_{1}, P_{2}, \ldots$ of monic polynomials over $F$ such that $I \in P_{r}$ for all $r$, let $Q$ be the set formed by those similarity classes of matrices over $F$ whose associated sequences of polynomials $\left(f_{1}(x), f_{2}(x) \ldots\right) \quad\left(\right.$ in the sense of $(3.1)$ ) satisfy $f_{p}(x) \in P_{r}$ for alz 
$r$. Then

$$
G_{Q}(t)=\prod_{r=1}^{\infty} g_{P_{r}}\left(t^{r}\right)
$$

Proof. It follows from (3.2) and the definition of $Q$ that

$$
G_{n}(Q)=\sum_{\substack{n_{1}, n_{2}, \ldots \geq 0 \\ \sum m_{p}=n}} g_{n_{1}}\left(P_{1}\right) g_{n_{2}}\left(P_{2}\right) \ldots
$$

which is equivalent to (4.22).

Proof of (4.3). If $M$ is the set of all monic polynomials in $F\left[x^{\delta}\right]$ which are not divisible by $x$, then

$$
\begin{aligned}
g_{M}(t) & =1+(q-1) t^{\delta}+(q-1) q t^{2 \delta}+\ldots \\
& =\left(1-t^{\delta}\right) /\left(1-q t^{\delta}\right) .
\end{aligned}
$$

Let us now choose $P_{1}, P_{2}, \ldots$ in the lemina as follows:

$$
P_{r}= \begin{cases}M & \text { if } d \mid r, \\ \{1\} & \text { otherwise. }\end{cases}
$$

Then, by (3.17) and (3.18), the resulting set $Q$ is the set $S_{\delta}^{d}$ of all GL-classes with $s$-index divisible by $d$ and $p$-index divisible by $\delta$. The Lemma gives

$$
G_{\delta} d^{(t)}=\prod_{\substack{r \\ d \mid r}} g_{M}\left(t^{r}\right)=c\left(t^{d \delta}\right),
$$

which is just another way of writing (4.3). This completes the proof.

Further preparation is needed for the proof of (4.15). We introduce certain unions of GL-classes within which the distribution of $G L$-classes according to determinant can be simply described.

Consider the sequence of polynomials (3.1) associated with a given $A \in G L_{n}$. Write each component polynomial down explicitly in the form: 
(4.23) $\quad f_{r}(x)=x^{n_{r}}+(-1)^{n_{r}-i} a_{r i} x^{i}+(-1)^{n_{r}-j} a_{r j} x^{j}+\ldots$,

where $n_{r}>i>j>\ldots$ and all coefficients $a_{r i}, a_{r j}, \ldots$ are non-zero. The index set

$$
\Omega_{p}(A)=\left\{n_{r}, i, j, \ldots\right\}
$$

is finite and non-empty with greatest and least members $n_{p}$ and 0 , and $\Omega_{r}(A)=\{0\}$ for almost all $r$. The sequence

$$
\text { (4.24) } \quad \Omega(A)=\left(\Omega_{1}(A), \Omega_{2}(A), \ldots\right)
$$

will be called the support of $A$. Since all matrices in the one $P G L$-class have the same support, we may speak of the support of a $P G L$-class or of any subset such as a GL-class.

Consider now the set $T(\Omega)$ of all matrices $A$ having a given support $(4.25)$

$$
\Omega=\left(\Omega_{1}, \Omega_{2}, \ldots\right) \text {, }
$$

where each $\Omega_{r}$ is a finite set such that $0 \in \Omega_{r}$ and where almost all (but not all) $\Omega_{r}$ are $\{0\}$. By (3.2), all matrices in $T(\Omega)$ have the same dimension, namely,

$$
n(\Omega)=\sum_{r} m_{p}(\Omega)
$$

where $n_{r}(\Omega)$ denotes the greatest member of $\Omega_{p}$. By (3.17) and (3.18) we have

$$
d(A)=(d(\Omega(A)), q-1), \delta(A)=(\delta(\Omega(A)), q-1),
$$

where $d(\Omega)$ denotes the greatest common divisor of the indices $r$ for which $\Omega_{p} \neq\{0\}$ and $\delta(\Omega)$ the greatest common divisor of the elements of $\Omega_{r}$

LEMMA. The GL-classes of matrices which make up $T(\Omega)$ can be parametrized by the elements of an abelian group $H(\Omega)$ in such a way that the mopping which assigns to each element of $H(\Omega)$ the determinant of the matrices in the corresponding GL-class is a group homomorphism mapping 
$H(\Omega)$ onto $\left(F^{*}\right)^{d(\Omega)}$

Proof. The $G L$-classes $(A)_{G L}, A \in T(\Omega)$, are already parametrized by the corresponding rows of polynomials (3.1). Replacing each $f_{p}(x)$ by the corresponding row of coefficients $\left(a_{r i}, a_{r j}, \ldots\right)$ (see (4.23)), we get a row of

$$
N(\Omega)=\sum_{r}\left(\left|\Omega_{r}\right|-1\right)
$$

non-zero elements of $F$, that is, an element of the direct product of $N(\Omega)$ copies of the group $F^{*}$. This direct product is the parameter group $H(\Omega)$. Let $r_{1}, \ldots, r_{s}$ be the indices $r$ for which $\operatorname{deg} f_{p}(x)>0$.

Then, by (3.3),

$$
\operatorname{det} A=\delta\left(f_{r_{1}}\right)^{r_{1}} \ldots \delta\left(f_{r_{s}}\right)^{r_{s}} \text {. }
$$

At the same time, by (4.23), the element of $H(\Omega)$ corresponding to $(A) G L$ has the form

$$
\left(\ldots, \delta\left(f_{r_{1}}\right), \ldots, \delta\left(f_{r_{2}}\right), \ldots, \delta\left(f_{r_{s}}\right), \ldots\right) .
$$

It follows that the mapping described in the lemma is indeed a homomorphism $H(\Omega) \rightarrow F^{*}$ and that the image of $H(\Omega)$ is

$$
\prod_{i=1}^{s}\left(F^{*}\right)^{r} i=\left(F^{*}\right)^{d(\Omega)} \text {. }
$$

This proves the lemma.

We are now in a position to prove (4.15). Let $D, E, \Delta, d, \delta$ be divisors of $q-I$ with $D E \ln (q-1)$. The set $(A)_{E ; G}$ in (4.13) with $G=P^{\Delta}$ becomes

$$
(A)_{E, \Delta}=\left\{\lambda T A T^{-1}: \lambda \in\left(F^{*}\right)_{E}, \operatorname{det} T \in\left(F^{*}\right)^{\Delta}\right\} .
$$

Let $T_{\delta}^{d}(n)$ denote the set of all matrices in $G L_{n}$ with s-index $d$ and $p$-index $\delta$. Then the numerical restriction $D E \mid n(q-1)$ guarantees that 
$T_{\delta}^{d}(n) \cap P^{D}(n)$ is a disjoint union of sets of the form $(A)_{E, \Delta}$, and (4.15) is equivalent to the assertion that the number of such sets is

$$
\frac{(d, D)(\delta, E)}{D E}(d, \Delta) c_{n}(d, \delta) .
$$

We shall prove this last result by showing that:

(a) if $A \in T_{\delta}^{d}(n)$ then $(A)_{E, \Delta}$ is a union of $d E /(d, \Delta)(\delta, E)$ SZ-classes;

(b) $T_{\delta}^{d}(n) \cap P(n)$ is a union of $d(d, D) c_{n}(d, \delta) / D$ SL-classes.

Proof of (a). The direct product $F^{*} \times F^{*}$ acts on the set of all $S L$-classes by the rule

$$
(\lambda, \mu) \circ(A)_{S L}=\left(\lambda T A T^{-1}\right)_{S L} \text {, where } \operatorname{det} T=\mu .
$$

The orbit of $(A)_{S L}$ consists of those $S L$-classes which make up $(A)_{P G L}$. Since $A \in T_{\delta}^{d}(n)$, it follows from (3.20) that the number of such $S L-c l a s s e s$ is $(q-1) d / \delta$. Since $\left(F^{*}\right)_{\delta} \times\left(F^{*}\right)^{d}$ is contained in the stabilizer. of $(A)_{S L}$ and has index $(q-1) d / \delta$ in $F^{*} \times F^{*}$, it must indeed be the stabilizer.

On the other hand, the $S L$-classes making up $(A)_{E, \Delta}$ form the orbit of $(A)_{S L}$ under the action of $\left(F^{*}\right)_{E} \times\left(F^{*}\right)^{\Delta}$. Therefore the number of $S L$-classes into which $(A)_{E, \Delta}$ splits is

$$
\begin{aligned}
\left|\left(F^{*}\right)_{E}:\left(F^{*}\right)_{E} \cap\left(F^{*}\right)_{\delta}\right|\left|\left(F^{*}\right)^{\Delta}:\left(F^{*}\right)^{\Delta} \cap\left(F^{*}\right)^{d}\right| & =(E /(\delta, E))(d /(d, \Delta)) \\
& =d E /(d, \Delta)(\delta, E) .
\end{aligned}
$$

Proof of (b). By (4.26) and (4.27), $T_{\delta}^{d}(n)$ is the disjoint union of those sets $T(\Omega)$ in the lemma which satisfy

$$
n(\Omega)=n,(d(\Omega), q-1)=d,(\delta(\Omega), q-1)=\delta .
$$

By that lemma, the proportion of $G L$-classes in such a $T(\Omega)$ having 
determinant in $\left(F^{*}\right)^{D}$ is

$$
\begin{aligned}
\left|\left(F^{*}\right)^{d(\Omega)}:\left(F^{*}\right)^{d(\Omega)} \cap\left(F^{*}\right)^{D}\right|^{-1} & =\left|\left(F^{*}\right)^{d}:\left(F^{*}\right)^{d} \cap\left(F^{*}\right)^{D}\right|^{-1} \\
& =(d, D) / D .
\end{aligned}
$$

Therefore the number of $G L$-classes in $T_{\delta}^{d}(n) \cap P(n)$ is $(d, D) c_{n}(d, \delta) / D$. On the other hand, by $(3.20)$, each GL-class in $T_{\delta}^{d}(n)$ splits into $d S L$-classes. It follows that $T_{\delta}^{d}(n) \cap P^{D}(n)$ splits into $d(d, D) c_{n}(d, \delta) / D S L$-classes, as we had to prove. The proof of (4.15) is now complete.

\section{References}

[1] Walter Feit and N.J. Fine, "Pairs of commuting matrices over a finite field", Duke Math. J. 27 (1960), 91-94.

[2] Nathan Jacobson, The theory of rings (American Mathematical Society Mathematical Surveys, 2. American Mathematical Society, New York, 1943).

[3] T.A. Ketter and G.1. Lehrer, "On conjugacy classes in certain isogenous groups", Bull. Austral. Math. Soc. 14 (1976), 371-377.

[4] G.I. Lehrer, "Characters, classes, and duality in isogenous groups", J. AZgebra 36 (1975), 278-286.

[5] I.G. Macdonald, "Numbers of conjugacy classes in some finite classical groups", Bulz. Austral. Math. Soc. 23 (1981), 23-48.

Department of Pure Mathematics,

University of Sydney,

Sydney,

New South Wales 2006,

Australia. 